\title{
Radial solutions with a vortex to an asymptotically linear elliptic equation
}

Tatsuya Watanabe

\begin{abstract}
In this paper, we study a two-dimensional nonlinear elliptic equation:

$$
-\Delta v(x)+V(x) v(x)=f(v(x)), x \in \mathbb{R}^{2}
$$

where $V(x)$ is radial, $V(x)$ behaves like $O\left(\frac{1}{|x|^{2}}\right)$ near zero and the nonlinearity $f$ is asymptotically linear at infinity. We show the existence of a nontrivial radial solution of (1.1) via the variational approach.
\end{abstract}

Mathematics Subject Classification (2000). Primary 35J20; Secondary 35 Q55.

Keywords. asymptotically linear nonlinearity, vortex, variational method.

\section{Introduction}

We consider the following elliptic equation in $\mathbb{R}^{2}$ :

$$
-\Delta v(x)+V(x) v(x)=f(v(x)) .
$$

In this paper, we study the case where $V(x)$ is radial, $V(x)$ behaves like $O\left(\frac{1}{|x|^{2}}\right)$ near zero and the nonlinearity $f$ is asymptotically linear at infinity. In this situation, we show the existence of a nontrivial radial solution of (1.1) which satisfies $v(x) \rightarrow 0$ as $|x| \rightarrow \infty$. As we will see later, a radial solution of (1.1) also satisfies $v(0)=0$ when $V(x)$ behaves like $O\left(\frac{1}{|x|^{2}}\right)$ in the two-dimensional problem.

Since we are looking for a radially symmetric solution, problem (1.1) leads to the following ordinary differential equation:

$$
-v^{\prime \prime}(r)-\frac{1}{r} v^{\prime}(r)+V(r) v(r)=f(v(r)), r \in(0, \infty) .
$$

Supported by Research Fellowships of the Japan Society for the Promotion of Science for Young Scientists 
To find a nontrivial (weak) solution of (1.1), we reduce (1.2) to another ordinary differential equation as in [20]. We put $v(r)=r^{-\frac{1}{2}} u(r)$. Then $u(r)$ satisfies

$$
-u^{\prime \prime}(r)+\left(V(r)-\frac{1}{4 r^{2}}\right) u(r)=r^{\frac{1}{2}} f\left(r^{-\frac{1}{2}} u(r)\right), r \in(0, \infty) .
$$

We find a nontrivial solution of (1.3) via the variational approach.

For the nonlinearity $f$, we assume

(f1) $f \in C\left(\mathbb{R}^{+}, \mathbb{R}\right) . f(s) \equiv 0$ for $s \leq 0$.

(f2) $\frac{f(s)}{s} \rightarrow 0$ as $s \rightarrow 0^{+}$.

(f3) There exists $c_{0} \in(0, \infty)$ such that $\frac{f(s)}{s} \rightarrow c_{0}$ as $s \rightarrow \infty$.

(f4) $\frac{f(s)}{s}$ is a non-decreasing function.

A typical example which satisfies (f1)-(f4) is given by $f(s)=\frac{c_{0} s^{3}}{1+s^{2}}$. For a potential $V(x)$, we assume

(V1) $V(x)=V(|x|), V(r) \in C(0, \infty)$.

(V2) There exists $V_{0}>0$ such that

$$
V(r)-\frac{1}{4 r^{2}} \geq V_{0} \text { for all } r \in(0, \infty) .
$$

(V3) There exist $\omega>\frac{1}{2}, r_{0}>0$ and $\tilde{V}(r) \in C[0, \infty)$ such that $V(r)$ can be written by the form $V(r)=\frac{\omega^{2}}{r^{2}}+\tilde{V}(r)$ for $0<r<r_{0}$.

Typical examples of $V(r)$ are $V(r)=1+\frac{\omega^{2}}{r^{2}}$ or $V(r)=r^{2}+\frac{\omega^{2}}{r^{2}}\left(V_{0}=\right.$ $2 \sqrt{\omega^{2}-\frac{1}{4}}$ in this case).

To state our main results, we define a function space $X$ by the closure of $C_{0}^{\infty}(0, \infty)$ with respect to the norm:

$$
\|u\|_{X}^{2}:=\int_{0}^{\infty}\left(u^{\prime}\right)^{2}+\left(V(r)-\frac{1}{4 r^{2}}\right) u^{2} d r .
$$

We also define

$$
\lambda:=\inf _{u \in X \backslash\{0\}} Q(u), Q(u):=\frac{\int_{0}^{\infty}\left(u^{\prime}\right)^{2}+\left(V(r)-\frac{1}{4 r^{2}}\right) u^{2} d r}{\int_{0}^{\infty} u^{2} d r}, u \in X \backslash\{0\} .
$$

From (V2), we have $Q(u) \geq V_{0}$ for all $u \in X \backslash\{0\}$. This implies $\lambda$ is well-defined and $\lambda \geq V_{0}$.

Theorem 1.1. Assume (f1)-(f4), (V1)-(V3) hold. Moreover we assume $c_{0}>\lambda$ where $c_{0}$ is defined in (f3). Then (1.1) has a nontrivial non-negative radial solution.

In the case $V(r)=r^{2}+\frac{\omega^{2}}{r^{2}}$, we can explicitly compute corresponding eigenvalues and eigenfunctions of the one-dimensional Schrödiger operator: $-\frac{d^{2}}{d r^{2}}+r^{2}+$ $\left(\omega^{2}-\frac{1}{4}\right) \frac{1}{r^{2}}$. Then we obtain the following corollary. 
Corollary 1.2. Let $V(r)=r^{2}+\frac{\omega^{2}}{r^{2}}$ and assume (f1)-(f4) hold. If $c_{0}>2(\omega+1)$, then (1.1) has a nontrivial non-negative radial solution.

Finally we can obtain the multiplicity result in the case $V(r)=r^{2}+\frac{\omega^{2}}{r^{2}}$.

Theorem 1.3. Let $V(r)=r^{2}+\frac{\omega^{2}}{r^{2}}$. Suppose (f2)-(f4) and (f5): $f \in C(\mathbb{R}, \mathbb{R})$ and $f(s)$ is an odd function.

Moreover we assume that $2(\omega-1+2 k)<c_{0}<2(\omega+1+2 k)$ for $k \in \mathbb{N}$. Then (1.1) has at least $k$ distinct pairs of nontrivial radial solutions.

Let us comment on the derivation of problem (1.1). We consider a nonlinear Schrödinger equation:

$$
i \frac{\partial \psi}{\partial t}=-\Delta \psi+K(x) \psi-f(\psi),
$$

where $\psi: \mathbb{R}^{N} \times \mathbb{R} \mapsto \mathbb{C}$ and $K: \mathbb{R}^{N} \mapsto \mathbb{R}$. A lot of works has been devoted in finding standing waves, that is, solutions of the form: $\psi(t, x)=\exp (-i E t) v(x)$ where $E$ is a real constant. Then $v(x)$ should satisfy problem (1.1) with $V(x)=K(x)-E$. Especially, problem (1.1) has been studied when $V(x) \in C\left(\mathbb{R}^{N}, \mathbb{R}\right)$ and the nonlinearity $f$ is asymptotically linear at infinity (see [3], [8], [12], [13] and references therein). In the physical point of view, asymptotically linear nonlinearity is said to have the saturation effect. This type of nonlinearities are important in the study of nonlinear optics (see [19] for related topics).

Recently in two-dimensional problem, another type of solutions of (1.4) has been studied. We consider a solution of (1.4) of the form: $\psi(t, x)=\exp (i \omega \theta+$ $i E t) v(r)$ where $(r, \theta)$ is a polar coordinate in $\mathbb{R}^{2}$ and $\omega \in \mathbb{N}$. Then we can see that problem (1.4) reduces to the equation:

$$
-\Delta v(x)+\left(K(x)+E+\frac{\omega^{2}}{|x|^{2}}\right) v(x)=f(v(x)), x \in \mathbb{R}^{2} .
$$

This kind of problems has been studied when the nonlinearity $f(s)$ is $|s|^{p-1} s$ or superlinear at infinity (see [2], [4], [5], [10]).

When $f(s)=-|s|^{2} s$, problem (1.1) appears in the study of the GrossPitaevskii theory of Bose-Einstein condensation. Especially a function $r^{2}+\frac{\omega^{2}}{r^{2}}$ appears if the Bose-Gas is confined in a harmonic trap potential and rotates around the origin (see [16], [17]). In this case, a constant $\omega$ means an eigenvalue of the angular momentum and a solution of (1.1) is called vortex state or $\omega$-vortex.

We should also mention that inverse square potentials are said to be Hardy type singular potentials in higher dimensional problems. Several papers are concerned with elliptic problems with this type of potentials (see e.g. [6], [7], [15], [18] and references therein).

The main features of this paper are the followings. Since the singularity $\frac{1}{|x|^{2}}$ is too strong in $\mathbb{R}^{2}$, we have to treat estimates near the origin carefully. Especially 
to find a weak solution of (1.1) (including the origin), we need decay estimates near the origin. We will show that if $v(x)$ is a nontrivial radial solution of (1.1), then $v(x)$ satisfies

$$
|v(x)| \leq C|x|^{\omega},|\nabla v(x)| \leq C|x|^{\omega-1},|x| \sim 0 .
$$

Then we will see that it is sufficient to find a nontrivial critical point of a functional which corresponds to (1.3) (see Proposition 3.2 below). Since we are looking for solutions which satisfy (1.1) including the origin, we are restricted to the radially symmetric case. It will be a future research to find a non-radial solution.

To obtain estimate (1.5), assumption (V3) (particularly $\omega>\frac{1}{2}$ ) plays an important role. In the original meaning, $\omega$ should be a natural number. However we can treat the case where $\omega$ is a general constant in the study of (1.1). The author believes that $\omega=\frac{1}{2}$ is a threshold for the existence of nontrivial solutions which satisfy (1.1) including the origin.

To prove Corollary 1.2 and Theorem 1.3, we have to compute eigenvalues and eigenfunctions of the corresponding Schrödinger operator. We will see that we can reduce the corresponding eigenvalue problem to a confluent hypergeometric equation when $V(r)=r^{2}+\frac{\omega^{2}}{r^{2}}$. Making use of confluent hypergeometric functions, we explicitly compute eigenvalues.

This paper is organized as follows. In section 2, we study basic properties of a function space $X$ and a corresponding Schrödinger operator. In section 3, we prove Theorem 1.1 by using the Mountain Pass Theorem. In section 4, we consider the case $V(r)=r^{2}+\frac{\omega^{2}}{r^{2}}$. We prove Theorem 1.3 by using the Linking Theorem.

\section{Preliminaries}

\subsection{Properties of the function space $X$}

In this subsection, we study basic properties of a function space which we will use in this paper. We define a function space $X$ by the closure of $C_{0}^{\infty}(0, \infty)$ with respect to the norm

$$
\|u\|_{X}=\int_{0}^{\infty}\left(u^{\prime}\right)^{2}+\left(V(r)-\frac{1}{4 r^{2}}\right) u^{2} d r
$$

We also define the inner product on $X$ by

$$
<u_{1}, u_{2}>_{X}:=\int_{0}^{\infty} u_{1}^{\prime} u_{2}^{\prime}+\left(V(r)-\frac{1}{4 r^{2}}\right) u_{1} u_{2} d r \text { for } u_{1}, u_{2} \in X
$$


From (V2), we have

$$
\int_{0}^{\infty}\left(u^{\prime}\right)^{2}+u^{2} d r \leq \frac{1}{\min \left\{1, V_{0}\right\}}\|u\|_{X}^{2}, \text { for } u \in X .
$$

This implies if $u \in X$, then

$$
u \in H_{0}^{1}(0, \infty)=\left\{u \in L^{2}(0, \infty) ; u^{\prime} \in L^{2}(0, \infty), u(0)=0\right\} .
$$

By one-dimensional Sobolev inequality, we have

$$
\|u\|_{L^{\infty}(0, \infty)} \leq C\|u\|_{X}, \lim _{r \rightarrow \infty} u(r)=0
$$

for some $C>0$.

Now for $u \in X$, we put $v(r)=r^{-\frac{1}{2}} u(r)$. Then we can see that

$$
\|u\|_{X}^{2}=\frac{1}{2 \pi} \int_{\mathbb{R}^{2}}|\nabla v|^{2}+V(x) v^{2} d x .
$$

From (V2), we have $V(x) \geq V_{0}+\frac{1}{4|x|^{2}}$. Thus it follows

$$
\int_{\mathbb{R}^{2}} v^{2} d x \leq \frac{2 \pi}{V_{0}}\|u\|_{X}^{2}, \int_{\mathbb{R}^{2}} \frac{v^{2}}{|x|^{2}} d x \leq 8 \pi\|u\|_{X}^{2} .
$$

This implies if $u \in X$, then $v(x)=|x|^{-\frac{1}{2}} u(x)$ satisfies

$$
v \in H^{1}\left(\mathbb{R}^{2}\right), v(x)=v(|x|) \text { and } \int_{\mathbb{R}^{2}} V(x) v^{2} d x<\infty .
$$

Next we study the behavior of $u \in X$ near the origin.

Lemma 2.1. Assume (V1)-(V2) hold. Then $u(r)$ satisfies $\lim _{r \rightarrow 0^{+}} r^{-\frac{1}{2}} u(r)=0$.

Proof. Since $v(r)=r^{-\frac{1}{2}} u(r)$, we show that $\lim _{r \rightarrow 0^{+}} v(r)=0$. For $0<a<b<1$, we have

$$
\begin{aligned}
\left|v(b)^{2}-v(a)^{2}\right|=\left|\int_{a}^{b} \frac{d}{d r}(v(r))^{2} d r\right|=\left|2 \int_{a}^{b} v v^{\prime} d r\right| \\
\leq C\left(\int_{a}^{b} \frac{v^{2}}{r} d r\right)^{\frac{1}{2}}\left(\int_{a}^{b} r\left(v^{\prime}\right)^{2} d r\right)^{\frac{1}{2}} \leq C\left(\int_{a \leq|x| \leq b} \frac{v^{2}}{|x|^{2}} d x\right)^{\frac{1}{2}}\left(\int_{a \leq|x| \leq b}|\nabla v|^{2} d x\right)^{\frac{1}{2}} \\
\leq C\left(\int_{|x| \leq b} \frac{v^{2}}{|x|^{2}} d x\right)^{\frac{1}{2}}\left(\int_{|x| \leq b}|\nabla v|^{2} d x\right)^{\text {for some } C>0 .}
\end{aligned}
$$


Since $|\nabla v|^{2}, \frac{v^{2}}{|x|^{2}} \in L^{1}\left(\mathbb{R}^{2}\right)$, it follows that $\left\{v(r)^{2}\right\}_{0<r<1}$ is a Cauchy-sequence as $r \rightarrow 0^{+}$. Thus there exists $\alpha \geq 0$ such that $\lim _{r \rightarrow 0^{+}} v(r)^{2}=\alpha$. On the other hand, since $\int_{\mathbb{R}^{2}} \frac{v^{2}}{|x|^{2}} d x<\infty$, it follows that $\alpha=0$. Thus we have

$$
\lim _{r \rightarrow 0^{+}} r^{-\frac{1}{2}} u(r)=\lim _{r \rightarrow 0^{+}} v(r)=0 .
$$

\subsection{Properties of one-dimensional Schrödinger operator L}

In this subsection, we correct basic properties of an operator which corresponds to problem (1.3). We define one-dimensional Schrödinger operator L by

$$
L:=-\frac{d^{2}}{d r^{2}}+\left(V(r)-\frac{1}{4 r^{2}}\right) \text { in } L^{2}(0, \infty), D(L)=\left\{H_{0}^{1}(0, \infty) ; L u \in L^{2}(0, \infty)\right\}
$$

For the self-adjointness of $L$, we have the following result.

Proposition 2.2. [14] (Weyl's limit point-limit circle criterion)

Suppose that a continuous real-valued function $W(r)$ on $(0, \infty)$ satisfies the followings. $W(r) \geq \frac{3}{4} r^{-2}$ near zero.

There exists $M(r) \in C^{1}(0, \infty)$ such that $M(r) \geq 0$ and

(i) $W(r) \geq-M(r)$ for all $r \in(0, \infty)$.

(ii) $\int_{1}^{\infty}(M(r))^{-\frac{1}{2}} d r=\infty$.

(iii) $\frac{M^{\prime}(r)}{(M(r))^{\frac{3}{2}}}$ is bounded near infinity.

Then $H:=-\frac{d^{2}}{d r^{2}}+W(r)$ is essentially self-adjoint on $C_{0}^{\infty}(0, \infty)$.

Lemma 2.3. Assume and (V1)-(V3) hold and $\omega$ (defined in (V3)) satisfies $\omega>1$. Then $L$ is an essentially self-adjoint operator on $C_{0}^{\infty}(0, \infty)$.

Proof. First we observe that from (V3)

$$
V(r)-\frac{1}{4 r^{2}}-\frac{3}{4 r^{2}}=\tilde{V}(r)+\frac{\omega^{2}-1}{r^{2}}, 0<r<r_{0}
$$

Since $\omega>1$ and $\tilde{V}(r)$ is bounded from below near zero, we have $V(r)-\frac{1}{4 r^{2}} \geq \frac{3}{4 r^{2}}$ near zero.

Next we choose $M(r)=r$. Then from (V2), we have

$$
V(r)-\frac{1}{4 r^{2}} \geq V_{0} \geq-r=-M(r) \text { for all } r>0 .
$$


Moreover,

$$
\int_{1}^{\infty} r^{-\frac{1}{2}} d r=+\infty, \frac{r^{\prime}}{r^{\frac{3}{2}}}=r^{-\frac{3}{2}} \rightarrow 0 \text { as } r \rightarrow \infty .
$$

Thus by Proposition 2.2, $L$ is essentially self-adjoint on $C_{0}^{\infty}(0, \infty)$.

Remark 2.4. (i) In general, let $H$ be a self-adjoint operator that is bounded from below. It is well-known that

$$
\inf _{u \in D(H),\|u\|=1}(u, H u)=\inf \sigma(H) .
$$

Then we can see that $\lambda=\inf \sigma(L)$ if $\omega>1$. When $\frac{1}{2}<\omega \leq 1, L$ is symmetric, positive definite but not necessarily self-adjoint. In this case, we can not readily see whether $\lambda=\inf \sigma(L)$ or not. We refer [9] for related topics.

(ii) Suppose $\omega>1$ and $V(r) \rightarrow \infty$ as $r \rightarrow \infty$. Then the spectrum of $L$ consists of discrete spectrums $\left\{\mu_{k}\right\}_{k=0}^{\infty}$ and the corresponding eigenfunction $\left\{\phi_{k}\right\}$ is an orthogonal complete system on $L^{2}(0, \infty)$.

(iii) If $\tilde{V}(r)$ in (V3) is non-negative near zero, the statement in Lemma 2.3 holds for $\omega=1$. Especially if $V(r)=r^{2}+\frac{\omega^{2}}{r^{2}}$ and $\omega \geq 1$, then $L$ is self-adjoint.

\section{Existence of a nontrivial solution}

In this section, we prove Theorem 1.1. Now we are looking for a nontrivial solution of the problem:

$$
-u^{\prime \prime}+\left(V(r)-\frac{1}{4 r^{2}}\right) u=r^{\frac{1}{2}} f\left(r^{-\frac{1}{2}} u\right) .
$$

For $u \in X$, we define an energy functional

$$
I(u):=\frac{1}{2}\|u\|_{X}^{2}-\int_{0}^{\infty} r F\left(r^{-\frac{1}{2}} u\right) d r, \text { where } F(s)=\int_{0}^{s} f(t) d t .
$$

Before we proceed to the proof of Theorem 1.1, we correct basic properties of the nonlinearity $f(s)$.

Suppose (f1)-(f3) hold. Then for all $\epsilon>0$, there exists $C_{\epsilon}>0$ such that

$$
|f(s)| \leq \epsilon|s|+C_{\epsilon}|s|^{p-1}, p \in(2, \infty) \text { for } s \geq 0 .
$$

Especially it follows

$$
|f(s)| \leq \epsilon|s|+C_{\epsilon}|s|^{5},|F(s)| \leq \frac{\epsilon}{2}|s|^{2}+\frac{C_{\epsilon}}{6}|s|^{6}, s \geq 0 .
$$


Next since $f(s)$ is asymptotically linear at infinity, there exists $M>0$ such that

$$
\frac{|f(s)|}{|s|} \leq M, \frac{|F(s)|}{s^{2}} \leq \frac{M}{2} \text { for all } s \geq 0
$$

Finally we assume (f4) additionally. Then

$$
\frac{2 F(s)}{s^{2}} \text { is a non - decreasing function. }
$$

First we show that if $u$ is a critical point of $I(u)$, then $v(x):=|x|^{-\frac{1}{2}} u(x)$ is a weak solution of problem (1.1). Since $\frac{1}{|x|^{2}} \notin L^{1}\left(\mathbb{R}^{2}\right)$, it needs some work.

Lemma 3.1. Assume (f1)-(f3), (V1)-(V3) hold. Suppose $u(r)$ is a nontrivial critical point of $I(u)$, then $u(r)$ satisfies

$$
|u(r)| \leq C r^{\frac{1}{2}+\omega},\left|u^{\prime}(r)\right| \leq C r^{\omega-\frac{1}{2}} \text { for } 0<r<r_{0}
$$

for some $C>0$ (which depends of $u$ ).

Proof. Since $u$ is a critical point of $I(u)$, it satisfies the following ordinary differential equation:

$$
-u^{\prime \prime}+\left(\omega^{2}-\frac{1}{4}\right) \frac{u}{r^{2}}=r^{\frac{1}{2}} f\left(r^{-\frac{1}{2}} u\right)-\tilde{V}(r) u=: G(r), 0<r<r_{0} .
$$

Now a general solution of the homogeneous linear equation $-u^{\prime \prime}+\left(\omega^{2}-\frac{1}{2}\right) \frac{u}{r^{2}}=0$ is given by $u(r)=A r^{\frac{1}{2}-\omega}+B r^{\frac{1}{2}+\omega}$ where $A$ and $B$ are arbitrary constants. Then the Wronskian of a pair $r^{\frac{1}{2}+\omega}, r^{\frac{1}{2}-\omega}$ is given by $W\left\{r^{\frac{1}{2}+\omega}, r^{\frac{1}{2}-\omega}\right\}=2 \omega$. Thus for $0<r<r_{0}$, a solution $u$ of (3.6) can be written by the form:

$$
u(r)=r^{\frac{1}{2}-\omega}\left(\frac{-1}{2 \omega} \int_{r}^{r_{0}} t^{\frac{1}{2}+\omega} G(t) d t+A\right)+r^{\frac{1}{2}+\omega}\left(\frac{1}{2 \omega} \int_{r}^{r_{0}} t^{\frac{1}{2}-\omega} G(t) d t+B\right) .
$$

Since $\omega>\frac{1}{2}$ and $u(0)=0$, we have

$$
\lim _{r \rightarrow 0^{+}}\left(\frac{-1}{2 \omega} \int_{r}^{r_{0}} t^{\frac{1}{2}+\omega} G(t) d t+A\right)=0 \text {, that is , } A=\frac{1}{2 \omega} \int_{0}^{r_{0}} t^{\frac{1}{2}+\omega} G(t) d t .
$$

Thus we obtain

$$
u(r)=\frac{r^{\frac{1}{2}-\omega}}{2 \omega} \int_{0}^{r} t^{\frac{1}{2}+\omega} G(t) d t+r^{\frac{1}{2}+\omega}\left(\frac{1}{2 \omega} \int_{r}^{r_{0}} t^{\frac{1}{2}-\omega} G(t) d t+B\right), 0<r<r_{0} .
$$


Since $G(r)=r^{\frac{1}{2}} f\left(r^{-\frac{1}{2}} u\right)-\tilde{V}(r) u$ and $\tilde{V}(r)$ is bounded in $\left[0, r_{0}\right]$, we have

$$
|G(r)| \leq r^{\frac{1}{2}}\left|f\left(r^{-\frac{1}{2}} u\right)\right|+\sup _{0 \leq r \leq r_{0}}|\tilde{V}(r)||u|
$$

Using (3.2), we obtain

$$
|G(r)| \leq r^{\frac{1}{2}}\left(\epsilon\left|r^{-\frac{1}{2}} u\right|+C_{\epsilon}\left|r^{-\frac{1}{2}} u\right|^{p-1}\right)+\sup _{0 \leq r \leq r_{0}}|\tilde{V}(r)||u| .
$$

By Lemma 2.1, we have $r^{-\frac{1}{2}} u(r) \rightarrow 0$ as $r \rightarrow 0^{+}$. Thus there exists $C>0$ such that

$$
\left|r^{-\frac{1}{2}} u(r)\right| \leq C \text { for } 0<r<r_{0}
$$

Therefore from (3.8), it follows $|G(r)| \leq C r^{\frac{1}{2}}, 0<r<r_{0}$. Then we have

$$
\begin{aligned}
& \left|\int_{0}^{r} t^{\frac{1}{2}+\omega} G(t) d t\right| \leq \int_{0}^{r} C t^{\frac{1}{2}+\omega+\frac{1}{2}} d t=\frac{C}{\omega+2} r^{\omega+2}, \\
& \left|\int_{r}^{r_{0}} t^{\frac{1}{2}-\omega} G(t) d t\right| \leq \int_{r}^{r_{0}} C t^{\frac{1}{2}-\omega+\frac{1}{2}} d t \leq C r^{2-\omega}+C .
\end{aligned}
$$

From (3.7), (3.9) and (3.10), we obtain

$$
|u(r)| \leq C\left(r^{\frac{1}{2}+\omega}+r^{\frac{1}{2}+2}\right), 0<r<r_{0} .
$$

If $\frac{1}{2} \leq \omega \leq 2$, we are done. Suppose $\omega>2$. Then $u(r) \leq C r^{\frac{1}{2}+2}$ for $0<r<r_{0}$. Using this estimate in (3.8), we have $|G(r)| \leq C r^{\frac{1}{2}+2}$. Then we obtain $|u(r)| \leq C\left(r^{\frac{1}{2}+\omega}+r^{\frac{1}{2}+4}\right)$. Continuing these procedures, we have $|u(r)| \leq C r^{\frac{1}{2}+\omega}$ for $0<r<r_{0}$.

Differentiating (3.7), we obtain $\left|u^{\prime}(r)\right| \leq C r^{-\frac{1}{2}+\omega}, 0<r<r_{0}$ in a similar way.

Proposition 3.2. Assume (V1)-(V3) hold. Let $u(r)$ be a critical point of $I(u)$. Then $v(x)=|x|^{-\frac{1}{2}} u(x)$ is a weak solution of

$$
-\Delta v+V(x) v=f(v) \text { in } \mathbb{R}^{2} .
$$

Proof. For $\phi \in C_{0}^{\infty}\left(\mathbb{R}^{2}\right)$, we show that

$$
\int_{\mathbb{R}^{2}} \nabla v \cdot \nabla \phi+V(x) v \phi d x=\int_{\mathbb{R}^{2}} f(v) \phi d x .
$$


Now since $u(r)$ is a critical point of $I(u), u(r)$ satisfies (3.1). Then $v(x)=$ $|x|^{-\frac{1}{2}} u(|x|)$ satisfies

$$
-\Delta v(x)+V(x) v(x)=f(v(x)) \text { a.e. } x \in \mathbb{R}^{2} \backslash\{|x| \geq \epsilon\}
$$

for all $\epsilon>0$. Multiplying $\phi$ and integrating over $|x| \geq \epsilon$, we have

$$
\int_{|x| \geq \epsilon}(-\Delta v+V(x) v-f(v)) \phi d x=0 .
$$

Next it follows that

$$
\begin{aligned}
& \int_{\mathbb{R}^{2}} \nabla v \cdot \nabla \phi d x=\lim _{\epsilon \rightarrow 0}\left\{-\int_{|x|=\epsilon} \nabla v \cdot \nu \phi d S-\int_{|x| \geq \epsilon} \Delta v \phi d x\right\}, \\
& \int_{\mathbb{R}^{2}} V(x) v \phi d x=\int_{|x|<\epsilon} V(x) v \phi d x+\int_{|x| \geq \epsilon} V(x) v \phi d x,
\end{aligned}
$$

where $\nu$ is an interior unit normal vector.

Now a direct computation shows that

$$
\frac{\partial v}{\partial x_{i}}=\frac{x_{i}}{r}\left(u^{\prime}-\frac{1}{2 r} u\right) r^{-\frac{1}{2}}, x \neq 0 .
$$

Then by Lemma 3.1, we have $|\nabla v \cdot \nu| \leq C \epsilon^{\omega-1}$ on $\{|x|=\epsilon\}$ if $\epsilon$ is small. Thus we obtain

$$
\int_{|x|=\epsilon} \nabla v \cdot \nu \phi d S \leq C \epsilon^{\omega} \max _{|x|=\epsilon}|\phi(x)| \rightarrow 0 \text { as } \epsilon \rightarrow 0
$$

and hence

$$
\int_{\mathbb{R}^{2}} \nabla v \cdot \nabla \phi d x=\lim _{\epsilon \rightarrow 0} \int_{|x| \geq \epsilon}-\Delta v \phi d x .
$$

Moreover from (V3), we have

$$
\begin{aligned}
& \int_{|x|<\epsilon} V(x) v \phi d x \leq \sup _{x \in \mathbb{R}^{2}}|\phi(x)| \int_{|x|<\epsilon} V(x)|v| d x \\
& \leq C \sup _{x \in \mathbb{R}^{2}}|\phi(x)| \int_{0}^{\epsilon}\left(\frac{\omega^{2}}{r^{2}}+\tilde{V}(r)\right) r^{\frac{1}{2}}|u| d r \leq C \sup _{x \in \mathbb{R}^{2}}|\phi(x)| \epsilon^{\omega} \rightarrow 0 \text { as } \epsilon \rightarrow 0 .
\end{aligned}
$$

Thus taking a limit $\epsilon \rightarrow 0$ in (3.11), we obtain

$$
\int_{\mathbb{R}^{2}} \nabla v \cdot \nabla \phi+V(x) v \phi d x=\int_{\mathbb{R}^{2}} f(v) \phi d x .
$$


To prove Theorem 1.1, it suffices to show the existence of a nontrivial critical point of $I(u)$ by Proposition 3.2. To this aim, we use the variant of Mountain Pass Theorem due to Bartolo, Benci and Fortunato [1].

Proposition 3.3. Let $E$ be a Hilbert space and consider a functional $J \in C(E, \mathbb{R})$ satisfying: $J(0)=0$,

(i) There exits $\rho>0, \alpha>0$ such that $J(u) \geq \alpha$ for all $u \in E,\|u\|=\rho$.

(ii) There exits $e \in E,\|e\|>\rho$ such that $J(e) \leq 0$.

(iii) For all $c>0$, every bounded sequence $\left\{u_{n}\right\} \subset E$ such that $J\left(u_{n}\right) \rightarrow c$, $J^{\prime}\left(u_{n}\right) \rightarrow 0$ in $E^{*}$ as $n \rightarrow \infty$, has a convergent subsequence.

(iv) For all $c>0$, there exist $\delta, R, \eta>0$ such that if $J(u) \in[c-\delta, c+\delta]$, $\|u\| \geq R$, then $\left\|J^{\prime}(u)\right\|\|u\| \geq \eta$.

Then there exists $u \in E$ such that $J(u) \geq \alpha$ and $J^{\prime}(u)=0$.

Hereafter in this section, we establish (i)-(iv) under assumptions (f1)-(f4), (V1)-(V3).

Lemma 3.4. Assume (f1)-(f3), (V1)-(V2) hold. Then there exists $\rho>0$ and $\alpha>0$ such that $I(u) \geq \alpha$ for all $u \in X,\|u\|_{X}=\rho$.

Proof. Now from (3.3), we have

$$
r F\left(r^{-\frac{1}{2}} u\right) \leq r\left(\frac{\epsilon}{2}\left(r^{-\frac{1}{2}} u\right)^{2}+\frac{C_{\epsilon}}{6}\left(r^{-\frac{1}{2}} u\right)^{6}\right)=\frac{\epsilon}{2} u^{2}+\frac{C_{\epsilon}}{6} \frac{u^{2}}{r^{2}} u^{4} .
$$

Thus from (2.1), we obtain

$$
\int_{0}^{\infty} r F\left(r^{-\frac{1}{2}} u\right) d r \leq \frac{\epsilon}{2}\|u\|_{L^{2}}+\frac{C_{\epsilon}}{6}\|u\|_{L^{\infty}}^{4} \int_{0}^{\infty} \frac{u^{2}}{r^{2}} d r \leq\left\{C \epsilon+C\|u\|_{X}^{4}\right\}\|u\|_{X}^{2}
$$

where $C$ is a positive constant which is independent of $u$. Thus it follows

$$
I(u) \geq \frac{1}{2}\|u\|_{X}^{2}-\left\{C \epsilon+C_{\epsilon}\|u\|_{X}^{4}\right\}\|u\|_{X}^{2}
$$

Choosing $\epsilon$ and $\|u\|_{X}=\rho$ small, we obtain $I(u) \geq \alpha$ for some $\alpha>0$.

Lemma 3.5. Assume (f1)-(f3), (V1)-(V2) hold. Moreover suppose $c_{0}>\lambda$. Then there exists $e \in X,\|e\|_{X}>\rho$ such that $I(e) \leq 0$. 
Proof. By the definition of $\lambda$ and from $c_{0}>\lambda$, there exists $u_{0} \in X$ such that $\lambda \leq Q\left(u_{0}\right)<c_{0}$. Especially we have

$$
\int_{0}^{\infty}\left(u_{0}^{\prime}\right)^{2}+\left(V(r)-\frac{1}{4 r^{2}}-c_{0}\right) u_{0}^{2} d r<0 .
$$

Now we claim that $\lim _{t \rightarrow \infty} \frac{I\left(t u_{0}\right)}{t^{2}}<0$. To this aim, we show

$$
\lim _{t \rightarrow \infty} \int_{0}^{\infty} \frac{r F\left(t r^{-\frac{1}{2}} u_{0}\right)}{t^{2}} d r=\frac{c_{0}}{2} \int_{0}^{\infty} u_{0}^{2} d r
$$

Now if $u_{0}(r) \neq 0$, we have

$$
\lim _{t \rightarrow \infty} \frac{r F\left(t r^{-\frac{1}{2}} u_{0}(r)\right)}{t^{2}}=\lim _{t \rightarrow \infty} \frac{F\left(t r^{-\frac{1}{2}} u_{0}\right)}{\left(t r^{-\frac{1}{2}} u_{0}\right)^{2}} u_{0}^{2}=\frac{c_{0}}{2} u_{0}^{2} .
$$

Moreover from (3.4), we have

$$
0 \leq \frac{r F\left(t r^{-\frac{1}{2}} u_{0}\right)}{t^{2}} \leq \frac{F\left(t r^{-\frac{1}{2}} u_{0}\right)}{\left(t r^{-\frac{1}{2}} u_{0}\right)^{2}} u_{0}^{2} \leq \frac{M}{2} u_{0}^{2} \text { a.e. } r \in(0, \infty)
$$

for all $t>0$. Thus by Lebesgue's dominated convergence theorem,

$$
\lim _{t \rightarrow \infty} \int_{0}^{\infty} \frac{r F\left(t r^{-\frac{1}{2}} u_{0}\right)}{t^{2}} d r=\frac{c_{0}}{2} \int_{0}^{\infty} u_{0}^{2} d r
$$

and

$$
\lim _{t \rightarrow \infty} \frac{I\left(t u_{0}\right)}{t^{2}}=\frac{1}{2} \int_{0}^{\infty}\left(u_{0}^{\prime}\right)^{2}+\left(V(r)-\frac{1}{4 r^{2}}-c_{0}\right) u_{0}^{2} d r<0 .
$$

Putting $e=t u_{0}$ for sufficiently large $t$, we have $I(e) \leq 0$.

Lemma 3.6. Assume (f1)-(f3) hold. For all $c>0$, let $\left\{u_{n}\right\} \subset X$ be a sequence such that $I\left(u_{n}\right) \rightarrow c, I^{\prime}\left(u_{n}\right) \rightarrow 0,\left\|u_{n}\right\|_{X}$ is bounded. Then $\left\{u_{n}\right\}$ has a convergent subsequence.

Proof. Since $\left\|u_{n}\right\|_{X}$ is bounded, we may assume that $u_{n} \rightarrow u$ in $X$ for some $u \in X$. Now for all $\phi \in X$, we have $I^{\prime}\left(u_{n}\right) \phi \rightarrow 0, I^{\prime}\left(u_{n}\right) u_{n} \rightarrow 0$. Thus we have

$$
\begin{aligned}
& \int_{0}^{\infty} u_{n}^{\prime} u^{\prime}+\left(V(r)-\frac{1}{4 r^{2}}\right) u_{n} u-r^{\frac{1}{2}} f\left(r^{-\frac{1}{2}} u_{n}\right) u d r=o(1), \\
& \left\|u_{n}\right\|_{X}^{2}-\int_{0}^{\infty} r^{\frac{1}{2}} f\left(r^{-\frac{1}{2}} u_{n}\right) u_{n} d r=o(1) .
\end{aligned}
$$


Since $u_{n} \rightarrow u$ in $X$, it follows

$$
\int_{0}^{\infty} u_{n}^{\prime} u^{\prime}+\left(V(r)-\frac{1}{4 r^{2}}\right) u_{n} u d r \rightarrow\|u\|_{X}^{2} .
$$

Thus we have

$$
\left\|u_{n}\right\|_{X}^{2}-\|u\|_{X}^{2}=\int_{0}^{\infty} r^{\frac{1}{2}} f\left(r^{-\frac{1}{2}} u_{n}\right)\left(u_{n}-u\right) d r+o(1) .
$$

Then it suffices to show that $\int_{0}^{\infty} r^{\frac{1}{2}} f\left(r^{-\frac{1}{2}} u_{n}\right)\left(u_{n}-u\right) d r \rightarrow 0$. We fix $R>0$ arbitrary. Then from (3.4),

$$
\begin{aligned}
& \int_{0}^{R}\left|r^{\frac{1}{2}} f\left(r^{-\frac{1}{2}} u_{n}\right)\left(u_{n}-u\right)\right| d r \\
& \quad \leq \int_{0}^{R}\left|\frac{f\left(r^{-\frac{1}{2}} u_{n}\right)}{r^{-\frac{1}{2}} u_{n}}\left\|u_{n}\right\| u_{n}-u\right| d r \leq M\left\|u_{n}\right\|_{X}\left(\int_{0}^{R}\left|u_{n}-u\right|^{2} d r\right)^{\frac{1}{2}} .
\end{aligned}
$$

Since $u_{n} \rightarrow u$ in $L_{l o c}^{2}(0, \infty)$, we have

$$
\lim _{n \rightarrow \infty} \int_{0}^{R} r^{\frac{1}{2}} f\left(r^{-\frac{1}{2}} u_{n}\right)\left(u_{n}-u\right) d r=0 .
$$

Next from (3.2), we have

$$
\begin{aligned}
& \int_{R}^{\infty} r^{\frac{1}{2}} f\left(r^{-\frac{1}{2}} u_{n}\right)\left(u_{n}-u\right) d r \\
& \quad \leq \epsilon \int_{R}^{\infty}\left|u_{n}\right|\left|u_{n}-u\right| d r+C_{\epsilon} \int_{R}^{\infty} r^{-\frac{1}{2}(p-2)}\left|u_{n}\right|^{p-1}\left|u_{n}-u\right| d r \\
& \quad \leq \epsilon\left\|u_{n}\right\|_{X}\left(\left\|u_{n}\right\|_{X}+\|u\|_{X}\right)+\frac{C_{\epsilon}}{R^{\frac{1}{2}(p-2)}}\left\|u_{n}\right\|_{X}^{p-1}\left(\left\|u_{n}\right\|_{X}+\|u\|_{X}\right) \\
& \leq C \epsilon+\frac{C_{\epsilon} C}{R^{\frac{1}{2}(p-2)}}
\end{aligned}
$$

for some $C>0$ independent of $\epsilon$ and $R$. Thus we obtain

$$
\limsup _{n \rightarrow \infty}\left|\int_{R}^{\infty} r^{\frac{1}{2}} f\left(r^{-\frac{1}{2}} u_{n}\right)\left(u_{n}-u\right) d r\right| \leq C \epsilon+\frac{C_{\epsilon} C}{R^{\frac{1}{2}(p-2)}} .
$$


Since $R$ is arbitrary and $C_{\epsilon}$ is independent of $R$, we have $\frac{C_{\epsilon} C}{R^{\frac{1}{2}(p-2)}} \rightarrow 0$ by taking a limit $R \rightarrow \infty$. Next passing a limit $\epsilon \rightarrow 0$, we obtain

$$
\lim _{n \rightarrow \infty} \int_{0}^{\infty} r^{\frac{1}{2}} f\left(r^{-\frac{1}{2}} u_{n}\right)\left(u_{n}-u\right) d r=0 .
$$

Lemma 3.7. Assume (f1)-(f4) hold. Moreover suppose $c_{0}>\lambda$. For all $c>0$, there exist $\delta, R, \eta>0$ such that if $c-\delta \leq I(u) \leq c+\delta$ and $\|u\|_{X} \geq R$, then $\left\|I^{\prime}(u)\right\|\|u\|_{X} \geq \eta$.

Proof. We fix $c>0$ arbitrary. For $n \in \mathbb{N}$, we put

$$
\begin{aligned}
m_{n}: & =\inf \left\{\left\|I^{\prime}(u)\right\|\|u\|_{X} ; u \in A_{n}\right\}, A_{n} \\
& =\left\{u \in X ; I(u) \in\left[c-\frac{1}{n}, c+\frac{1}{n}\right],\|u\|_{X} \geq n\right\} .
\end{aligned}
$$

Here we define $m_{n}=+\infty$ if $A_{n}=\emptyset$. We show that $m_{n}>0$ for some $n \in \mathbb{N}$.

Now we suppose by contradiction that $m_{n}=0$ for every $n \in \mathbb{N}$. Then there exists $u_{n} \in A_{n}$ such that

$$
I\left(u_{n}\right) \rightarrow c,\left\|u_{n}\right\|_{X} \rightarrow \infty \text { as } n \rightarrow \infty,\left\|I^{\prime}\left(u_{n}\right)\right\|\left\|u_{n}\right\|_{X}<\frac{1}{n} .
$$

We divide the proof into four steps.

Step 1: For all $t>0$ and $n \in \mathbb{N}$, it follows

$$
I\left(t u_{n}\right) \leq \frac{1+t^{2}}{2 n}+I\left(u_{n}\right) \text { for all } t>0, n \in \mathbb{N} .
$$

From (3.12), we have

$$
-\frac{1}{n}<\left\|u_{n}\right\|_{X}^{2}-\int_{0}^{\infty} r^{\frac{1}{2}} f\left(r^{-\frac{1}{2}} u_{n}\right) u_{n} d r<\frac{1}{n} .
$$

We claim that for all $t>0$ and $n \in \mathbb{N}$,

$$
I\left(t u_{n}\right) \leq \frac{t^{2}}{2 n}+\int_{0}^{\infty} \frac{1}{2} r^{\frac{1}{2}} f\left(r^{-\frac{1}{2}} u_{n}\right) u_{n}-r F\left(r^{-\frac{1}{2}} u_{n}\right) d r .
$$

We put

$$
G(t):=\frac{1}{2} t^{2} r^{\frac{1}{2}} f\left(r^{-\frac{1}{2}} u_{n}\right) u_{n}-r F\left(t r^{-\frac{1}{2}} u_{n}\right)
$$


Then

$$
G^{\prime}(t)=t r^{\frac{1}{2}} f\left(r^{-\frac{1}{2}} u_{n}\right) u_{n}-r^{\frac{1}{2}} u_{n} f\left(t r^{-\frac{1}{2}} u_{n}\right)=t\left(\frac{f\left(r^{-\frac{1}{2}} u_{n}\right)}{r^{-\frac{1}{2}} u_{n}}-\frac{f\left(t r^{-\frac{1}{2}} u_{n}\right)}{t r^{-\frac{1}{2}} u_{n}}\right) u_{n}^{2}
$$

Since $\frac{f(s)}{s}$ is a non-decreasing function, we have $G(t) \leq G(1)$ for all $t>0$. Now

$$
\begin{aligned}
I\left(t u_{n}\right) & =\frac{t^{2}}{2}\left\|u_{n}\right\|_{X}^{2}-\int_{0}^{\infty} r F\left(t r^{-\frac{1}{2}} u_{n}\right) d r<\frac{t^{2}}{2 n}+\int_{0}^{\infty} G(t) d r \leq \frac{t^{2}}{2 n}+\int_{0}^{\infty} G(1) d r \\
& =\frac{t^{2}}{2 n}+\int_{0}^{\infty} \frac{1}{2} r^{\frac{1}{2}} f\left(r^{-\frac{1}{2}} u_{n}\right) u_{n}-r F\left(r^{-\frac{1}{2}} u_{n}\right) d r
\end{aligned}
$$

On the other hand, from (3.14),

$$
\begin{gathered}
\left.-\frac{1}{2 n}+\int_{0}^{\infty} \frac{1}{2} r^{\frac{1}{2}} f\left(r^{-\frac{1}{2}} u_{n}\right) u_{n}\right)-r F\left(r^{-\frac{1}{2}} u_{n}\right) d r \\
\leq I\left(u_{n}\right)=\frac{1}{2}\left\|u_{n}\right\|_{X}^{2}-\int_{0}^{\infty} r F\left(r^{-\frac{1}{2}} u_{n}\right) d r .
\end{gathered}
$$

Thus

$$
\int_{0}^{\infty} \frac{1}{2} r^{\frac{1}{2}} f\left(r^{-\frac{1}{2}} u_{n}\right) u_{n}-r F\left(r^{-\frac{1}{2}} u_{n}\right) d r \leq I\left(u_{n}\right)+\frac{1}{2 n}
$$

Substituting (3.16) into (3.15), we obtain

$$
I\left(t u_{n}\right) \leq \frac{1+t^{2}}{2 n}+I\left(u_{n}\right) \text { for all } t>0, n \in \mathbb{N} .
$$

Next we define $w_{n}:=\frac{2 c^{\frac{1}{2}} u_{n}}{\left\|u_{n}\right\|_{X}}$. Then we may assume that $w_{n} \rightarrow w$ in $X$ for some $w \in X$.

Step 2: $w(r) \not \equiv 0$.

We suppose by contradiction that $w(r) \equiv 0$. Then for any $R>0, w_{n}(r) \rightarrow 0$ a.e. $r \in(0, R)$. First we show that $\int_{0}^{\infty} r F\left(r^{-\frac{1}{2}} w_{n}\right) d r \rightarrow 0$ as $n \rightarrow \infty$. Now from (3.5), $\frac{2 F(s)}{s^{2}}$ is a non-decreasing function. Since $\left\|w_{n}\right\|_{L^{\infty}} \leq M\left\|w_{n}\right\|_{X}=M 2 c^{\frac{1}{2}}$ for some $M>0$, we have

$$
\begin{aligned}
\int_{R}^{\infty} r F\left(r^{-\frac{1}{2}} w_{n}\right) d r & =\int_{R}^{\infty} \frac{r\left(r^{-\frac{1}{2}} w_{n}\right)^{2}}{2} \times \frac{2 F\left(r^{-\frac{1}{2}} w_{n}\right)}{\left(r^{-\frac{1}{2}} w_{n}\right)^{2}} d r \\
& \leq \int_{R}^{\infty} \frac{w_{n}^{2}}{2} \times \frac{2 F\left(r^{-\frac{1}{2}} M 2 c^{\frac{1}{2}}\right)}{\left(r^{-\frac{1}{2}} M 2 c^{\frac{1}{2}}\right)^{2}} d r \leq C \sup _{r \in[R, \infty)} \frac{F\left(r^{-\frac{1}{2}} M 2 c^{\frac{1}{2}}\right)}{\left(r^{-\frac{1}{2}} M 2 c^{\frac{1}{2}}\right)^{2}}
\end{aligned}
$$


From (f2), we have $\frac{F(s)}{s^{2}} \rightarrow 0$ as $s \rightarrow 0^{+}$. Thus for all $\epsilon>0$, there exists $R_{\epsilon}>0$ such that

$$
\sup _{r \in\left[R_{\epsilon}, \infty\right)} \frac{F\left(r^{-\frac{1}{2}} M 2 c^{\frac{1}{2}}\right)}{\left(r^{-\frac{1}{2}} M 2 c^{\frac{1}{2}}\right)^{2}}<\epsilon .
$$

On the other hand, from (3.4), it follows

$$
\int_{0}^{R_{\epsilon}} r F\left(r^{-\frac{1}{2}} w_{n}\right) d r \leq C \int_{0}^{R_{\epsilon}} w_{n}^{2} d r
$$

Thus

$$
\limsup _{n \rightarrow \infty}\left|\int_{0}^{\infty} r F\left(r^{-\frac{1}{2}} w_{n}\right) d r\right| \leq \epsilon
$$

Since $\epsilon$ is arbitrary, we obtain $\int_{0}^{\infty} r F\left(r^{-\frac{1}{2}} w_{n}\right) d r \rightarrow 0$ as $n \rightarrow \infty$.

Now by the definition of $w_{n}$ and the above estimate, we have

$$
I\left(w_{n}\right)=\frac{1}{2}\left\|w_{n}\right\|_{X}^{2}-\int_{0}^{\infty} r F\left(r^{-\frac{1}{2}} w_{n}\right) d r=2 c+o(1) .
$$

We put $t_{n}=\frac{2 \sqrt{c}}{\left\|u_{n}\right\|}$. Then $t_{n} \rightarrow 0$ as $n \rightarrow \infty$. Moreover from (3.13),

$$
2 c+o(1)=I\left(w_{n}\right)=I\left(t_{n} u_{n}\right) \leq \frac{1+t_{n}^{2}}{2 n}+I\left(u_{n}\right)=c+o(1) .
$$

This is a contradiction. Thus we have $w(r) \not \equiv 0$.

Step 3: $w(r)$ satisfies

$$
\int_{0}^{\infty} w^{\prime} \phi^{\prime}+\left(V(r)-\frac{1}{4 r^{2}}\right) w \phi-c_{0} w \phi d r=0 \text { for all } \phi \in X .
$$

First we show that $w(r) \geq 0$. In fact, since $I^{\prime}\left(u_{n}\right) \rightarrow 0$, we have $I^{\prime}\left(u_{n}\right) w_{n}^{-} \rightarrow$ 0 where $w_{n}^{-}(r)=\max \left\{0,-w_{n}(r)\right\}$. Then from $f(s) \equiv 0$ for $s \leq 0$, we obtain $\left\|w_{n}^{-}\right\|_{X}^{2} \rightarrow 0$ and hence $w(r) \geq 0$ a.e. $r \in(0, \infty)$. Now we fix $\phi \in X$ arbitrary. Then

$$
\left|I^{\prime}\left(u_{n}\right) \phi\right| \leq\left\|I^{\prime}\left(u_{n}\right)\right\|\left\|u_{n}\right\|_{X} \times \frac{\|\phi\|_{X}}{\left\|u_{n}\right\|_{X}} \leq \frac{\|\phi\|_{X}}{n\left\|u_{n}\right\|_{X}} .
$$

On the other hand since

$$
I^{\prime}\left(u_{n}\right) \phi=<u_{n}, \phi>_{X}-\int_{0}^{\infty} r^{\frac{1}{2}} f\left(r^{-\frac{1}{2}} u_{n}\right) \phi d r
$$


we have

$$
\frac{2 c^{\frac{1}{2}} I^{\prime}\left(u_{n}\right) \phi}{\left\|u_{n}\right\|}=<w_{n}, \phi>_{X}-2 c^{\frac{1}{2}} \int_{0}^{\infty} \frac{r^{\frac{1}{2}} f\left(r^{-\frac{1}{2}} u_{n}\right) \phi}{\left\|u_{n}\right\|_{X}} d r .
$$

We claim that

$$
\int_{0}^{\infty} \frac{2 c^{\frac{1}{2}} r^{\frac{1}{2}} f\left(r^{-\frac{1}{2}} u_{n}\right)}{\left\|u_{n}\right\|_{X}} \phi d r \rightarrow \int_{0}^{\infty} c_{0} w \phi d r
$$

as $n \rightarrow \infty$. Now we can see that

$$
\frac{2 c^{\frac{1}{2}}}{\left\|u_{n}\right\|_{X}} r^{\frac{1}{2}} f\left(r^{-\frac{1}{2}} u_{n}\right)=\frac{f\left(r^{-\frac{1}{2}} u_{n}\right)}{r^{-\frac{1}{2}} u_{n}} \times w_{n} .
$$

Since $u_{n}(r)=\frac{\left\|u_{n}\right\|_{X}}{c^{\frac{1}{2}}} w_{n}(r)$ and $w(r) \not \equiv 0$, we have $u_{n}(r) \rightarrow \infty$ a.e. $r \in(0, \infty)$ as $n \rightarrow \infty$. Now we may assume that $\phi \in C_{0}^{\infty}(0, \infty)$ and supp $\phi \subset(a, b)$ for some $0<a<b<\infty$. Since $w_{n}(r) \rightarrow w(r)$ in $L^{2}(a, b)$, there exists $h(r) \in L^{1}(a, b)$ such that $\left|w_{n}(r)\right| \leq h(r)$ a.e. $r \in(a, b)$. Combining with (3.4), we can apply Lebesgue's dominated convergence theorem to obtain (3.19). Taking a limit in (3.18), we obtain (3.17).

Step 4: If $c_{0}>\lambda$, then there is no nontrivial non-negative function which satisfies (3.17).

First we fix $\Lambda$ such that $\lambda<\Lambda<c_{0}$. By the definition of $\lambda$, there exists $v \in X$ so that $Q(v)<\Lambda$. Then we may assume that $v \in C_{0}^{\infty}(0, \infty)$. We choose $0<a<b<\infty$ so that supp $v \subset(a, b)$.

For $u \in H_{0}^{1}(a, b) \backslash\{0\}$, we define

$$
\tilde{Q}(u):=\frac{\int_{a}^{b}\left(u^{\prime}\right)^{2}+\left(V(r)-\frac{1}{4 r^{2}}\right) u^{2} d r}{\int_{a}^{b} u^{2} d r} .
$$

Since $V(r)-\frac{1}{4 r^{2}}$ is bounded on $[a, b], \tilde{\lambda}:=\inf _{u \in H_{0}^{1}(a, b) \backslash\{0\}} \tilde{Q}(u)$ is attained. Then

$$
\tilde{\lambda} \leq \tilde{Q}(v) \leq Q(v)<\Lambda<c_{0}
$$

On the other hand, let $\tilde{u} \in H_{0}^{1}(a, b)$ be a minimizer of $\tilde{Q}(u)$. Then we may assume that $\tilde{u}(r) \geq 0$ and $\tilde{u}$ satisfies

$$
-\tilde{u}^{\prime \prime}+\left(V(r)-\frac{1}{4 r^{2}}\right) \tilde{u}=\tilde{\lambda} \tilde{u}, r \in(a, b) .
$$


Moreover by Hopf's Lemma and Maximum Principle, it follows $\tilde{u}^{\prime}(a)>0, \tilde{u}^{\prime}(b)$ $<0$ and $\tilde{u}(r)>0$. Now

$$
\begin{aligned}
\tilde{\lambda} \int_{a}^{b} w \tilde{u} d r & =\int_{a}^{b} w\left(-\tilde{u}^{\prime \prime}+\left(V(r)-\frac{1}{4 r^{2}}\right) \tilde{u}\right) d r \\
& =\int_{a}^{b} w^{\prime} \tilde{u}^{\prime}+\left(V(r)-\frac{1}{4 r^{2}}\right) w \tilde{u} d r-w(b) \tilde{u}^{\prime}(b)+w(a) \tilde{u}^{\prime}(a) \\
& \geq \int_{a}^{b} w^{\prime} \tilde{u}^{\prime}+\left(V(r)-\frac{1}{4 r^{2}}\right) w \tilde{u} d r=\int_{a}^{b}\left(-w^{\prime \prime}+\left(V(r)-\frac{1}{4 r^{2}}\right) w\right) \tilde{u} d r \\
& +w^{\prime}(b) \tilde{u}(b)-w^{\prime}(a) \tilde{u}(a)=c_{0} \int_{a}^{b} w \tilde{u} d r .
\end{aligned}
$$

Since $w(r) \geq 0$ and $\tilde{u}(r)>0$, this implies $\tilde{\lambda} \geq c_{0}$. This contradicts to (3.20).

By step 1-4, we obtain $m_{n}>0$ for some $n \in \mathbb{N}$.

By Lemma 3.4-3.7, we can apply Proposition 3.3. Since $f(s) \equiv 0$ for $s \leq 0$, a nontrivial critical point $u(r)$ of $I(u)$ satisfies $u(r) \geq 0$ for all $r \geq 0$. By Maximum Principle, we have $u(r)>0$ for all $r \in(0, \infty)$. Together with Proposition 3.2, we obtain Theorem 1.1.

\section{The special case $V(r)=r^{2}+\frac{\omega^{2}}{r^{2}}$}

In this section, we consider the case $V(r)=r^{2}+\frac{\omega^{2}}{r^{2}}$. First we consider the following eigenvalue problem:

$$
-U^{\prime \prime}+\left(r^{2}+\left(\omega^{2}-\frac{1}{4}\right) \frac{1}{r^{2}}\right) U=\mu U, r \in(0, \infty)
$$

with the boundary conditions $U(0)=0$ and $U(r) \rightarrow 0$ as $r \rightarrow \infty$.

Using confluent hypergeometric functions, we can explicitly obtain eigenvalues and eigenfunctions of (4.1). For basic properties of confluent hypergeometric functions, we refer [11] chapter 9.

Lemma 4.1. Eigenvalues $\mu_{k}$ and eigenfunctions $\psi_{k}(r)$ of problem (4.1) are given by

$$
\mu_{k}=2(\omega+1+2 k), k \in \mathbb{N} \cup\{0\}, \phi_{k}(r)=e^{-\frac{r^{2}}{2}} r^{\omega+\frac{1}{2}} \sum_{j=0}^{k} \frac{(-1)^{j}}{(\omega+1)_{j}} r^{2 j},
$$

where $(p)_{j}=\frac{\Gamma(p+j)}{\Gamma(p)}=p(p+1) \cdots(p+j-1),(p)_{0}=1$. 
Proof. For a solution $U(r)$ of (4.1), we put $U(r)=e^{-\frac{r^{2}}{2}} r^{\omega+\frac{1}{2}} z\left(r^{2}\right)$. A direct computation shows that a function $z(r)$ should satisfy the following equation:

$$
r z^{\prime \prime}+(\omega+1-r) z^{\prime}-\left(\frac{2 \omega+2-\mu}{4}\right) z=0, r \in(0, \infty) .
$$

We write $\alpha:=\frac{2 \omega+2-\mu}{4}, \gamma:=1+\omega$ for simplicity. Then $z(r)$ satisfies so-called confluent hypergeometric equation of the form:

$$
r z^{\prime \prime}+(\gamma-r) z^{\prime}-\alpha z=0 .
$$

Our purpose is to choose $\mu$ so that $U(r)=e^{-\frac{r^{2}}{2}} r^{\omega+\frac{1}{2}} z\left(r^{2}\right)$ satisfies the two boundary conditions. For this purpose, we have to give general solutions of (4.2).

If $\gamma \neq 0,-1,-2, \cdots$, a function

$$
\Phi(\alpha, \gamma ; r):=\sum_{j=0}^{\infty} \frac{(\alpha)_{j}}{(\gamma)_{j}} \frac{r^{j}}{j !}
$$

satisfies (4.2). Function $\Phi$ is said to be a confluent hypergeometric function. Especially if $\alpha=-k$ for some $k \in \mathbb{N} \cup\{0\}$, then $(\alpha)_{k+1}=0$. This implies

$$
\Phi(-k, \gamma ; r)=\sum_{j=0}^{\infty} \frac{(-k)_{j}}{(\gamma)_{j}} \frac{r^{j}}{j !}=\sum_{j=0}^{k} \frac{(-1)^{j}}{(\gamma)_{j}} \frac{k !}{(k-j) ! j !} r^{j}
$$

We suppose now that $\alpha \neq-k$ for all $k \in \mathbb{N} \cup\{0\}$.

If $\gamma \notin \mathbb{N}$, then $r^{1-\gamma} \Phi(1+\alpha-\gamma, 2-\gamma ; r)$ is also a solution of (4.2) and it is linearly independent of $\Phi(\alpha, \gamma ; r)$.

If $\gamma=n_{0}+1$ for some $n_{0} \in \mathbb{N} \cup\{0\}$, then the linearly independent solution is given by $\Psi\left(\alpha, n_{0}+1 ; r\right)$

$$
\begin{aligned}
= & \frac{(-1)^{n_{0}+1}}{\Gamma\left(\alpha-n_{0}\right)} \sum_{j=0}^{\infty} \frac{(\alpha)_{j}}{\left(n_{0}+j\right) !} \frac{r^{j}}{j !}\left(\psi(\alpha+j)-\psi(1+j)-\psi\left(n_{0}+1+j\right)+\log r\right) \\
& +\frac{1}{\Gamma(\alpha)} \sum_{j=0}^{n_{0}-1} \frac{(-1)^{j}\left(n_{0}-j-1\right) !\left(\alpha-n_{0}\right)_{j}}{j !} r^{j-n_{0}},
\end{aligned}
$$

where $\psi(p)=\frac{\Gamma^{\prime}(p)}{\Gamma(p)}$ and we set $\sum_{j=0}^{-1}$ is zero. Function $\Psi$ is called a confluent hypergeometric function of the second kind.

Then by the definitions, we can see that

$$
\begin{aligned}
\Phi(\alpha, \gamma ; r) & =1+O(r), r \sim 0, \\
r^{1-\gamma} \Phi(1+\alpha-\gamma, 2-\gamma ; r) & =r^{1-\gamma}+o\left(r^{1-\gamma}\right), r \sim 0, \\
\Psi\left(\alpha, n_{0}+1 ; r\right) & =\frac{\left(n_{0}-1\right) !}{\Gamma(\alpha)} r^{-n_{0}}+o\left(r^{-n_{0}}\right), r \sim 0 .
\end{aligned}
$$


Moreover it is known that

$$
\begin{aligned}
& \Phi(\alpha, \gamma ; r) \sim e^{r} r^{-(\gamma-\alpha)}, r \sim \infty \\
& \quad r^{1-\gamma} \Phi(1+\alpha-\gamma, 2-\gamma ; r) \sim e^{r} r^{-(\gamma-\alpha)}, r \sim \infty \\
& \Psi\left(\alpha, n_{0}+1 ; r\right) \sim r^{-\alpha}, r \sim \infty
\end{aligned}
$$

Next we claim that if $\alpha \neq-k$ for all $k \in \mathbb{N} \cup\{0\}$, then there is no nontrivial solution of (4.1) which satisfies boundary conditions $U(0)=0$ and $U(r) \rightarrow 0$ as $r \rightarrow \infty$.

We suppose $\alpha \neq-k$ for all $k \in \mathbb{N} \cup\{0\}$ and $\gamma=n_{0}+1, n_{0} \in \mathbb{N} \cup\{0\}$. Then $U(r)$ can be written by the form:

$$
U(r)=A e^{-\frac{r^{2}}{2}} r^{\omega+\frac{1}{2}} \Phi\left(\alpha, \gamma ; r^{2}\right)+B e^{-\frac{r^{2}}{2}} r^{\omega+\frac{1}{2}} \Psi\left(\alpha ; \gamma ; r^{2}\right)
$$

where $A, B$ are constants.

Since $r^{\omega+\frac{1}{2}} \Psi\left(\alpha, \gamma ; r^{2}\right) \sim r^{-\omega+\frac{1}{2}}, r \sim 0$ and $\omega>\frac{1}{2}, U(0)=0$ implies $B=0$. On the other hand, since

$$
e^{-\frac{r^{2}}{2}} r^{\omega+\frac{1}{2}} \Phi\left(\alpha, \gamma ; r^{2}\right) \sim e^{-\frac{r^{2}}{2}} r^{\omega+\frac{1}{2}} e^{r^{2}} r^{-2(\gamma-\alpha)} \rightarrow \infty \text { as } r \rightarrow \infty
$$

$U(r) \rightarrow 0$ as $r \rightarrow \infty$ implies $A=0$. Thus $U(r) \equiv 0$. Arguing similarly, we obtain the same conclusion in the case $\gamma \notin \mathbb{N} \cup\{0\}$. We define

From above, we obtain $\alpha=-k$, that is, $\mu=2(\omega+1+2 k)$ for $k \in \mathbb{N} \cup\{0\}$.

$$
\phi_{k}(r):=e^{-\frac{r^{2}}{2}} r^{\omega+\frac{1}{2}} \sum_{j=0}^{k} \frac{(-1)^{j}}{(\gamma)_{j}} \frac{k !}{(k-j) ! j !} r^{2 j}
$$

Then we can see that $\phi_{k}(r)$ satisfies (4.1). Moreover $\phi_{k}(0)=0$ and $\lim _{r \rightarrow \infty} \phi_{k}(r)=0$. This implies $\phi_{k}(r)$ is an eigenfunction of (4.1) corresponding to the eigenvalue $\mu_{k}=2(\omega+1+2 k)$.

When $V(r)=r^{2}+\frac{\omega^{2}}{r^{2}}$, we can easily see that $\lambda$ is achieved because the embedding $X \hookrightarrow L^{2}(0, \infty)$ is compact. Then by Lemma 4.1 , we have $\lambda=2(\omega+1)$ for $V(r)=r^{2}+\frac{\omega^{2}}{r^{2}}$. Thus we obtain Corollary 1.2.

Next we put $\psi_{k}(r):=\frac{\phi_{k}(r)}{\left\|\phi_{k}\right\|_{L^{2}(0, \infty)}}$. By Remark 2.4, we have already known the completeness of $\left\{\psi_{k}\right\}$ in the case $\omega \geq 1$. Our next purpose is to show that $\left\{\psi_{k}\right\}$ is an orthonormal complete system even if $\frac{1}{2}<\omega<1$ by using the explicit expressions of $\left\{\psi_{k}\right\}$.

Lemma 4.2. $\left\{\psi_{k}(r)\right\}_{k=0}^{\infty}$ is an orthonormal complete system on $L^{2}(0, \infty)$. 
Proof. Now we can easily see that $\int_{0}^{\infty} \psi_{j^{\prime}} \psi_{j} d r=0$ for $j^{\prime} \neq j$. Next we assume that there exists $u \in L^{2}(0, \infty)$ such that $\int_{0}^{\infty} u(r) \psi_{k}(r) d r=0$ for all $k \in \mathbb{N} \cup\{0\}$. We show that $u(r)=0$ a.e. $r \in(0, \infty)$. This implies $\left\{\psi_{k}(r)\right\}$ is an orthonormal complete system.

Now by the definition of $\psi_{k}(r)$, it follows

$$
\psi_{k}(r)=\psi_{k-1}(r)+e^{-\frac{r^{2}}{2}} r^{\omega+\frac{1}{2}} \frac{(-1)^{k}}{(\gamma)_{k}} r^{2 k} .
$$

Since $\int_{0}^{\infty} u \psi_{k} d r=0$ for all $k \in \mathbb{N} \cup\{0\}$, we obtain

$$
\int_{0}^{\infty} u(r) e^{-\frac{r^{2}}{2}} \frac{(-1)^{k}}{(1+\omega)_{k}} r^{\omega+\frac{1}{2}+2 k} d r=0 \text { for all } k \in \mathbb{N} \cup\{0\} .
$$

Especially for $k \in \mathbb{N} \cup\{0\}$,

$$
\int_{0}^{\infty} u(r) e^{-\frac{r^{2}}{2}} r^{\omega+\frac{1}{2}} r^{2 k} d r=0
$$

Now we put $v(r):=u(r) e^{-\frac{r^{2}}{2}} r^{\omega+\frac{1}{2}}$ and define $\tilde{v}(r)=v(r)$ for $r \geq 0,=v(-r)$ for $r<0$. Then $\tilde{v}(r)$ is an even function, $\tilde{v} \in L^{2}(\mathbb{R})$ and from (4.3), it satisfies

$$
\int_{-\infty}^{\infty} \tilde{v}(r) r^{2 k} d r=0 \text { for all } k \in \mathbb{N} \cup\{0\} .
$$

For $z \in \mathbb{C}$, we define $G(z):=\int_{\mathbb{R}} \tilde{v}(r) e^{-i z r} d r$. Then $G(z)$ is well-defined for $z \in \mathbb{C}$ and analytic in $z$. For $k \in \mathbb{N}$, we have $G^{(k)}(0)=(-i)^{k} \int_{\mathbb{R}} \tilde{v}(r) r^{k} d r$. From (4.4), we have $G^{(2 k)}(0)=0$. On the other hand, since $\tilde{v}(r)$ is even and $r^{2 k+1}$ is odd, we have

$$
\int_{\mathbb{R}} \tilde{v}(r) r^{2 k+1} d r=0 \text { for all } k \in \mathbb{N} \cup\{0\} .
$$

Hence $G^{(k)}(0)=0$ for all $k \in \mathbb{N} \cup\{0\}$. Since $G(z)$ is analytic, this implies $G(z) \equiv 0$. On the other hand, $G(z)$ is a Fourier transform of $\frac{\tilde{v}(r)}{(2 \pi)^{\frac{1}{2}}}$, we have $\tilde{v}(r)=0$ a.e. $r \in \mathbb{R}$. Thus $u(r) e^{-\frac{r^{2}}{2}} r^{\omega+\frac{1}{2}}=0$ and hence $u(r)=0$ a.e. $r \in(0, \infty)$.

To prove Theorem 1.3, we use a variant of Linking Theorem due to Bartolo, Benci and Fortunato [1].

Proposition 4.3. Let $H$ be a Hilbert space and suppose that $J \in C^{1}(H, \mathbb{R})$ satisfies: 
(i) $J(0) \geq 0$.

(ii) $J$ is even.

(iii) For all $c>0$, every bounded sequence $\left\{u_{n}\right\} \subset E$ such that $J\left(u_{n}\right) \rightarrow c$, $J^{\prime}\left(u_{n}\right) \rightarrow 0$ in $E^{*}$ as $n \rightarrow \infty$, has a convergent subsequence.

(iv) For all $c \in(0, \infty)$, there exist $\sigma, R, \alpha>0$ such that if $[c-\sigma, c+\sigma] \subset(0, \infty)$, $J(u) \in[c-\sigma, c+\sigma]$ and $\|u\| \geq R$, then $\left\|J^{\prime}(u)\right\|\|u\| \geq \alpha$.

(v) There exist two closed subspaces $H^{+}, H^{-}$with codim $H^{+}<\infty$ and constants $J(0)<c_{1}<c_{2}, \rho>0$ such that

$$
J(u) \geq c_{1}, u \in H^{+} \cap\{u \in H ;\|u\|=\rho\}, J(u)<c_{2}, u \in H^{-} .
$$

If $\operatorname{dim} H^{-} \geq \operatorname{codim} H^{+}$, then $J$ has at least $n=\operatorname{dim} H^{-}-\operatorname{codim} H^{+}$distinct pairs of critical points whose critical values belong to $\left[c_{1}, c_{2}\right]$.

Proof of Theoreom 1.3. We assume (f2)-(f5) and $2(\omega-1+2 k)<c_{0}<$ $2(\omega+1+2 k)$ for $k \in \mathbb{N}$. By assumptions (f2) and (f5), (i), (ii) of Proposition 4.3 are clear. Moreover we have already proved (iii) by Lemma 3.6.

Next we show (iv). Arguing similarly as Lemma 3.7, we can see that there exists $w(r) \in X, w(r) \not \equiv 0$ such that

$$
\int_{0}^{\infty} w^{\prime} \phi^{\prime}+\left(r^{2}+\left(\omega^{2}-\frac{1}{4}\right) \frac{1}{r^{2}}\right) w \phi d r=c_{0} \int_{0}^{\infty} w \phi d r
$$

for all $\phi \in X$. The difference to the proof of Lemma 3.7 is that we can not obtain $w(r) \geq 0$. However by Lemma 4.1 , we have $c_{0}$ should satisfy $c_{0}=2\left(\omega+1+2 k_{0}\right)$ for $k_{0} \in \mathbb{N} \cup\{0\}$. This contradicts to the assumption $2(\omega-1+2 k)<c_{0}<2(\omega+1+2 k)$, $k \in \mathbb{N}$. Thus (iv) is satisfied.

It remains to show (v). First we set suitable two closed subspaces. For eigenvalue $\mu_{k}$ of problem (4.1), we denote $M_{k}$ by the corresponding eigenspace. For given $k \in \mathbb{N}$, we define

$$
H^{-}:=\oplus_{0 \leq j \leq k-1} M_{j}, H^{+}:=\overline{\operatorname{span}\left\{\psi_{j}\right\}_{j \geq 0}} .
$$

Then it follows that $\operatorname{dim} H^{-}=k$. Moreover by Lemma 4.2 , we have codim $H^{+}=0$.

Now we can see that there exist $c_{1}, \rho>0$ such that if $u \in H^{+},\|u\|_{X}=\rho$, then $I(u) \geq c_{1}$. The proof is similar to that of Lemma 3.4.

Next we show that there exists $c_{2}>c_{1}$ such that $I(u)<c_{2}$ for $u \in H^{-}$. First we claim that for all $\delta>0$, there exists $R>0$ such that $I(u) \leq \delta, u \in H^{-}$, $\|u\|_{X} \geq R$.

Now we fix $u \in H^{-}$and consider $t u$ for $t>0$. Then $t u \in H^{-}$and

$$
I(t u)=\frac{t^{2}}{2}\|u\|_{X}^{2}-\int_{0}^{\infty} r F\left(r^{-\frac{1}{2}} t u\right) d r .
$$


By the proof of Lemma 3.5, we have

$$
\frac{1}{t^{2}} \int_{0}^{\infty} r F\left(r^{-\frac{1}{2}} t u\right) d r \rightarrow \frac{c_{0}}{2} \int_{0}^{\infty} u^{2} d r \text { as } t \rightarrow \infty .
$$

On the other hand, for $u \in H^{-}, u$ can be written by the form $u=\sum_{j=0}^{k-1} c_{j} \psi_{j}$, $c_{j} \in \mathbb{R}$. Then

$$
\|u\|_{X}^{2}=\sum_{j=0}^{k-1} \mu_{j} c_{j}^{2}\left\|\psi_{j}\right\|_{L^{2}(0, \infty)}^{2} \leq \mu_{k-1}\|u\|_{L^{2}(0, \infty)}^{2} .
$$

Thus we have

$$
\frac{I(t u)}{t^{2}} \rightarrow \frac{1}{2}\left(\mu_{k}-c_{0}\right)\|u\|_{L^{2}(0, \infty)}^{2} \text { as } t \rightarrow \infty .
$$

By the assumption $c_{0}>2(\omega-1+2 k)=\mu_{k-1}$, we have $\frac{I\left(t_{0} u\right)}{t_{0}^{2}}<0$ for sufficiently large $t_{0}$. Putting $v=t_{0} u$, we have $I(v) \leq \delta$ for all $\delta>0$.

Next we fix $\delta>c_{1}$ arbitrary. Then there exists $R>0$ such that if $u \in H^{-}$, $\|u\|_{X} \geq R$, then $I(u) \leq \delta$.

We put

$$
K:=\sup \left\{I(u) ; u \in H^{-},\|u\|_{X} \leq R\right\}
$$

Then we can see that $K<\infty$. Putting $c_{2}:=\max \{\delta, K\}$, we have $I(u) \leq c_{2}$ for all $u \in H^{-}$. Thus (v) is satisfied.

From above, we can apply Proposition 4.3. Since $\operatorname{dim} H^{-}-\operatorname{codim} H^{+}=k$, we obtain $k$ distinct pairs of critical points.

Remark 4.4. Suppose $\omega>1$ and $V(r) \rightarrow \infty$ as $r \rightarrow \infty$. Then by Remark 2.4, the same conclusion as Theorem 1.3 holds if $c_{0}$ lies in the $k$-th spectral gap of $L$, that is, $\mu_{k-1}<c_{0}<\mu_{k}$.

\section{Acknowledgement}

The author would like to thank the referees for reading the manuscript carefully.

\section{References}

[1] P. Bartolo, V. Benci and D. Fortunato, Abstract critical point theorems and applications to some nonlinear problems with strong resonance at infinity. Nonlinear Anal. TMA. 7 (1983), 981-1012. 
[2] V. Benci and T. D'Aprile, The semiclassical limit of the nonlinear Schrödinger equation in a radial potential. J. Diff. Eqns. 184 (2002), 109-138.

[3] D. G. Costa and H. Tehrani, On a class of asymptotically linear elliptic problems in $\mathbb{R}^{N}$. JDE 173 (2001), 470-494.

[4] T. D'Aprile, Some existence and concentration results for nonlinear Schrödinger equations. Comm. Pure Appl. Anal. 1 (2002), 457-474.

[5] T. D'Aprile, On a class of solutions with non-vanishing angular momentum for nonlinear Schrödinger equations. Diff. Int. Eqns. 16 (2003), 349-384.

[6] V. Felli, E. Marchini and S. Terracini, On Schrödinger operators with multipolar inverse-square potentials, preprint.

[7] V. Felli and S. Terracini, Nonlinear Schrödinger equations with symmetric multi-polar potentials. Calc. Var. PDE. 27 (2006), 25-58.

[8] J. Jeanjean and K. Tanaka, A positive solution for an asymptotically linear elliptic problem on $\mathbb{R}^{N}$ autonomous at infinity. ESIAM Control Optim. Calc. Var. 7 (2002), 597-614.

[9] H. Kalf, U.-W. Schmincke, J. Walter and R. Wüst, On the spectral theory of Schrödinger and Dirac operators with strongly singular potentials, Spectral theory and differential equations (Proc. Sympos. Dundee, 1974; dedicated to Konrad Jörgens), pp. 182-226. Lect. Notes in Math. Vol. 448, Springer Berlin, 1975.

[10] K. Kurata and T. Watanabe, A remark on asymptotic profiles of radial solutions with a vortex to a nonlinear Schrödinger equation. Comm. Pure Appl. Anal. 5 (2006), 597-610.

[11] N. N. Lebedev, Special functions and their applications. Revised edition, translated from the Russian and edited by Richard A. Silverman. Unabridged and corrected republication. Dover Publications, Inc., New York, 1972.

[12] G. Li and H. S. Zhou, The existence of a positive solution to asymptotically linear scalar field equations. Proc. Royal Soc. Edin. 130(A) (2000), 81-105.

[13] Z. Liu and Z. Q. Wang, Existence of a positive solution of an elliptic equation on $\mathbb{R}^{N}$. Proc. Royal Soc. Edin. 134(A) (2004), 191-200.

[14] M. Reed and B. Simon, Methods of modern mathematical physics. II, Fourier analysis, self-adjointness. Academic Press, Harcourt Brace Jovanovich, Publishers, New York-London, 1975.

[15] D. Ruiz and M. Willem, Elliptic problems with critical exponents and Hardy potentials. J. Diff. Eqns. 190 (2003), 524-538.

[16] R. Seiringer, Gross-Pitaevskii theory of the rotating Bose gas. Comm. Math. Phys, 229 (2002), 491-509.

[17] R. Seiringer, Symmetry breaking in a model of a rotating Bose gas. Contemp. Math. 307 (2002), 281-286,

[18] D. Smets, Nonlinear Schrödinger equations with Hardy potential and critical nonlinearities. Trans. AMS. 357 (2005), 2909-2938. 
[19] C. A. Stuart, Guidance properties of nonlinear planar waveguides. Arch. Rat. Mech. Anal. 125 (1993), 145-200.

[20] C. A. Stuart and H. S. Zhou, Applying the Mountain Pass theorem to an asymptotically linear elliptic equation on $\mathbb{R}^{N}$. Comm. PDE, 24 (1999), 1731-1758.

Tatsuya Watanabe

Department of Mathematics

School of Science and Engineering

Waseda University

3-4-1 Ohkubo

Shinjuku-ku

Tokyo 169-8555

Japan

email: tatsuw@kurenai.waseda.jp

Received: 6 September 2007

Accepted: 14 January 2008

Published Online First 03 October 2008

To access this journal online:

http://www.birkhauser.ch 
